\title{
Outcomes of Antiretroviral Treatment in Programmes with and without Routine Viral Load Monitoring in Southern Africa
}

\author{
Olivia Keiser ${ }^{1}$, Benjamin H. Chi ${ }^{2}$, Thomas Gsponer ${ }^{1}$, Andrew Boulle ${ }^{3}$, Catherine Orrell ${ }^{4}$, \\ Sam Phiri ${ }^{5}$, Nicola Maxwell ${ }^{3}$, Mhairi Maskew ${ }^{6}$, Hans Prozesky ${ }^{7}$, Matthew P Fox ${ }^{6,8,9}$, Andrew \\ Westfall $^{2}$, Matthias Egger ${ }^{1}$, and for the leDEA Southern Africa Collaboration
}

${ }^{1}$ Division of International and Environmental Health, Institute of Social and Preventive Medicine (ISPM), University of Bern, Bern, Switzerland ${ }^{2}$ Centre for Infectious Disease Research in Zambia, Lusaka, Zambia ${ }^{3}$ School of Public Health and Family Medicine, University of Cape Town, South Africa ${ }^{4}$ The Desmond Tutu HIV Centre, Institute of Infectious Disease and Molecular Medicine, University of Cape Town, Cape Town, South Africa ${ }^{5}$ Lighthouse Trust Clinic, Kamuzu Central Hospital, Lilongwe, Malawi ${ }^{6}$ Clinical HIV Research Unit, Department of Medicine, Faculty of Health Sciences, University of Witwatersrand, Johannesburg, South Africa ${ }^{7}$ Division of Infectious Diseases, Department of Medicine, University of Stellenbosch and Tygerberg Academic Hospital, Cape Town, South Africa ${ }^{8}$ Center for Global Health and Development, Boston University, Boston MA, USA ${ }^{9}$ Department of Epidemiology, Boston University, Boston MA, USA

\section{Abstract}

Objectives-To compare outcomes of antiretroviral therapy (ART) in South Africa, where viral load monitoring is routine, with Malawi and Zambia, where monitoring is based on CD4 cell counts.

\begin{abstract}
Methods-We included 18,706 adult patients starting ART in South Africa and 80,937 patients starting in Zambia or Malawi. We examined CD4 responses in models for repeated measures, and the probability of switching to second-line regimens, mortality and loss to follow-up in multi-state models, measuring time from six months.

Findings-In South Africa 9.8\% (9.1-10.5\%) had switched at 3 years, $1.3 \%$ (95\% CI 0.9-1.6\%) remained on failing first-line regimens, $9.2 \%(8.5-9.8 \%)$ were lost to follow-up and 4.3\% (3.9$4.8 \%$ ) had died. In Malawi and Zambia more patients were on a failing first-line regimen (3.7\%, 3.6-3.9\%), fewer patients had switched $(2.1 \%, 2.0-2.3 \%)$ and more patients were lost (15.3\%, $15.0-15.6 \%)$ or had died $(6.3 \%, 6.0-6.5 \%)$. Median CD4 cell counts were lower in South Africa at start of ART (93 vs. 132 cells $/ \mu \mathrm{L}, \mathrm{p}<0.001)$ but higher after 3 years $(425$ vs. 383 cells $/ \mu \mathrm{L}$, $\mathrm{p}<0.001)$. The hazard ratio comparing South Africa with Malawi and Zambia, adjusted for age, sex, first-line regimen and CD4 cell count, was 0.58 (95\% CI 0.50-0.66) for death and $0.53(0.48-$ 0.58 ) for loss to follow-up.
\end{abstract}

Conclusions-Over 3 years of ART mortality was lower in South Africa than in Malawi or Zambia. The more favourable outcome in South Africa might be explained by viral load

Correspondence to: Olivia Keiser, Institute of Social and Preventive Medicine (ISPM), University of Bern, Finkenhubelweg 11, CH-3012 Bern, Switzerland. Tel.:+41 3163135 15; Fax: +41 3163135 20; okeiser@ ispm.unibe.ch.

Author contributions

O.K., T.G. and M.E. designed the study. O.K. and T.G. performed the statistical analyses. O.K. and M.E. wrote the first draft of the manuscript. All authors contributed to the interpretation of the results and to the final version of the manuscript. B.H.C., A.B., C.O., S.P., N.M., M.M., H.P., M.P.F, A.W. were involved in data acquisition and data management. O.K. had full access to all of the data in the study and takes responsibility for the integrity of the data and the accuracy of the data analysis. M.E. and A.B. are the principle investigators of IeDEA Southern Africa. 
monitoring leading to earlier detection of treatment failure, adherence counselling and timelier switching to second-line ART.

\section{INTRODUCTION}

The World Health Organization (WHO) estimates that over 5 million HIV-1 infected people were receiving antiretroviral therapy (ART) in low- and middle-income countries by the end of 2009 [1]. As access to HIV treatment continues to expand, more people are experiencing treatment failure, and switching to second-line therapy is on the increase. Viral load monitoring is typically not available in the public-sector in resource-limited settings: viral load monitoring is expensive and the necessary laboratory infrastructure is difficult to implement and maintain, particularly in rural areas.

In the absence of viral load monitoring, diagnosis of treatment failure relies on immunologic (i.e. CD4 cell counts) and clinical criteria [2]. The ability of CD4 counts to predict virologic failure is, however, limited: sensitivity and positive predictive value of the immunological WHO criteria for virological treatment failure have been shown to be poor [3] [4]. Use of these criteria may therefore lead to unnecessary switching to second-line ART among patients with suppressed viral replication, or cause undue delays in switching among patients with real - but undetected - virological failure.

In a recent analysis of 17 ART programmes in resource-limited settings we found that patients with access to viral load monitoring were more likely to switch to second-line therapy earlier and at higher CD4 cell counts than those enrolled in programmes without viral load monitoring [5]. Delays in switching will increase the time on low CD4 cell counts, and may promote the selection of resistant strains and thus affect long-term prognosis. In the present study we analyzed data from treatment programmes in Southern Africa to compare switching to second-line ART, loss to follow-up and mortality in the Republic of South Africa (RSA), where viral load monitoring is routine, to outcomes in Zambia and Malawi, where monitoring is based on CD4 cell counts.

\section{METHODS}

\section{Antiretroviral treatment programmes}

The International epidemiological Databases to Evaluate AIDS in Southern Africa (IeDEASA) is a collaboration of ART programmes in Southern Africa [6]. Data are collected at ART initiation (baseline) and each follow-up visit, using standardized instruments, and transferred in regular intervals to data centres at the Universities of Cape Town, RSA and Bern, Switzerland. All sites have ethical approval to collect data and participate in IeDEASA.

We included four public-sector ART programmes from RSA, which monitor viral load and CD4 cell counts every 3 to 6 months: Khayelitsha [7], Gugulethu [8] and the Tygerberg clinic [9] in Cape Town, and the Themba Lethu clinic [10] in Johannesburg. The South African programmes ('viral load sites') were compared with two treatment programmes from Malawi and Zambia, which monitor CD4 cell counts but have only limited access to viral load measurements (also referred to as 'non viral load sites'): the Lighthouse clinic at Kamuzu Central Hospital in Lilongwe [11] and the Ministry of Health - Centre for Infectious Disease Research in Zambia (MoH-CIDRZ) programme in Lusaka [12, 13]. All six programmes trace patients lost to follow-up. In Khayelitsha, patients lost to follow-up who could not be contacted by telephone are visited at home by a clinic nurse. In Gugulethu, patients are allocated a therapeutic counsellor who lives in the same community, visits patients at home and provides counselling and adherence support. In Themba Lethu and 
Tygerberg patients are traced using mobile telephone contacts. The Lighthouse clinic and the MoH-CIDRZ programme use community health workers to trace patients.

\section{Eligibility criteria and definitions}

We included all treatment naïve patients aged 16 years and older who started ART with a non-nucleoside reverse transcriptase inhibitor (NNRTI)-based regimen and who had at least one day of follow-up. We defined immunological failure as a decline in the CD4 cell count to the baseline value or below, a decline of at least $50 \%$ from the highest count on treatment or a persistent CD 4 cell count below 100 cells $/ \mu \mathrm{L}$ after six months of antiretroviral therapy [2]. Virological failure was defined as a plasma HIV viral load value above 10,000 copies/ $\mathrm{ml}[2]$. For both immunologic and virologic failure we required two consecutive values within 12 months of each other meeting the criteria. For immunological failure the second value had to be equal to or lower than the first.

A switch to a second-line ART regimen was defined as a change from the initial regimen to a protease inhibitor (PI)-based regimen after at least six months of follow-up with a simultaneous change in at least one nucleoside reverse transcriptase inhibitor (NRTI). Clinical disease stage was defined as less advanced (CDC stage A/B or WHO stage I/II) or advanced (CDC stage $\mathrm{C}$ or WHO stage III/IV). A patient was considered lost to follow-up (LTFU) if the last visit was more than 12 months before the closure date for that site, with the closure date defined as the most recent visit date recorded in the database for that site. Only patients with at least 12 potential months of follow-up could therefore be lost to follow-up.

\section{Analyses of treatment outcomes}

We plotted Kaplan-Meier curves to determine the probability of death from all causes, measuring time from ART initiation. Differences between viral load and non-viral load patients were compared using log-rank tests. We then built a multi-state model [14] to compare probabilities of death, loss to follow-up and switching to second-line regimens between viral load and non viral load programmes, measuring time from ART initiation. After 6 months of ART, when treatment response is assessed, a patient may remain on a non-failing first-line regimen or move to treatment failure (virological failure in sites with routine viral load monitoring or immunologic failure in sites without routine viral load monitoring), second-line ART, loss to follow-up or death (Figure 1). A patient failing firstline therapy may remain on the failing regimen, switch to second-line ART, be lost to follow-up or die. A patient switching may remain on the new regimen, be lost to follow-up or die. Death and loss to follow-up are final (absorbing) states in the model [14].

We calculated hazard ratios (HRs) separately for the first 6 months of ART, and from 6 months to the end of follow-up. We present HRs for death and loss to follow-up, and for the second period additionally for switching to second-line ART. We compared sites with and without viral load monitoring, adjusting for age (per 10 years increase), sex, first-line regimen, CD4 cell count (per 100 cell increase) and WHO clinical stage (WHO stage III and IV versus stage I and II) at ART initiation in the first analysis, and for age, sex, first-line regimen and CD4 cell count at 6 months in the second analysis. Four categories of first line regimens were included in the model: lamivudine, stavudine and nevirapine (3TC/d4T/ NVP); lamivudine, zidovudine and nevirapine (3TC/ZDV/NVP); lamivudine, stavudine and efavirenz (3TC/d4T/EFV) and lamivudine, zidovudine and efavirenz (3TC/ZDV/EFV) or other. We imputed missing CD4 cell counts at baseline and 6 months, and missing WHO clinical stage at baseline. Imputations were based on multinomial and linear regression models with disease stage and CD4 count as dependent variables and site, age, sex, year of starting ART, time to death and CD4 cell count (in case of missing clinical stage) or clinical 
stage (in case of missing CD4 count) as independent variables. Analyses were run on each of 20 datasets; results were combined with Rubin's rules [15]. In sensitivity analyses we repeated the analyses dropping one cohort in turn and restricting analyses to patients with complete data (complete-case analysis).

\section{Analysis of CD4 cell counts over time}

We used models for repeated measures to compare CD4 cell count trajectories between sites with and without viral load monitoring. We constructed a multi-level model (CD4 measurements within patients) with a random intercept and slope for each patient, transforming time to the square root and the natural logarithm of time (fractional polynomial $[2,(-0.5, \ln )])$, as described previously [16]. All patients with a baseline CD4 cell count and at least one additional CD4 count were included. We again examined the influence of single cohorts by repeating the analyses dropping one cohort in turn.

\section{Non-HIV related mortality}

We explored to what extent differences in HIV-free background mortality influenced results. We obtained estimates of non-HIV-related mortality for the year 2004 (the last year for which data were available) from the Global Burden of Disease Study [17][18]. We compared the expected HIV-unrelated mortality between countries with and without viral load monitoring. Briefly, the expected number of deaths due to causes other than HIV between months 7 and 36 was calculated by multiplying the number of person-years by the corresponding sex, age- (in 5-year age groups) and country-specific rates of HIV-free mortality.

Data were analyzed using Stata software version 11 (College Station, Texas, USA) and R version 2.10 (The R Development Core Team).

\section{RESULTS}

\section{Patient and programme characteristics}

A total of 99,643 patients met eligibility criteria and were included: 7,230 patients from Khayelitsha, 2,658 patients from Gugulethu, 7,457 patients from Themba Lethu, 1,361 patients from Tygerberg, 9,604 patients from Lighthouse and 71,333 patients from $\mathrm{MoH}-$ CIDRZ. Overall 18,706 patients were treated at sites with routine viral load monitoring in RSA ( 27,288 person-years of follow-up) and 80,937 patients at sites without access to viral load monitoring in Malawi and Zambia (147,876 person-years of follow-up). The proportion of patients excluded because they were only seen once and had no follow-up was similar across sites: $1.5 \%$ (range across sites $0.4-2.2 \%$ ) in viral load sites and $1.3 \%$ (range 1.0$3.6 \%)$ in non viral load sites.

Patients from viral load sites were more likely to be female (66\% versus $62 \%)$ and had lower CD4 cell counts ( 93 versus 132 cells $/ \mu \mathrm{L}$ ) at start of therapy (Table 1 ). In both settings most patients started ART with a regimen that combined 3TC/d4T either with NVP or EFV. AZT, didanosine (ddI) and boosted lopinavir (LPV/r) was the most common second-line regimen in viral load sites whereas in CD4 sites a combination of tenofovir (TDF), emtricitabine (FTC) and LPV/r was most commonly used. The South African programmes had better access to diagnostic examinations and treatments than the programmes in Malawi and Zambia, including availability of computer tomography, Cryptococcus neoformans antigen testing, and first-line therapy for opportunistic infections. Further details on patient characteristics and availability of diagnostic examinations are given in Webtable 1 and Webtable 2 . 


\section{Treatment outcomes}

Of the eligible 99,643 patients, 3,009 (3.0\%) failed their first-line regimen, 1,839 (1.8\%), switched to a second-line regimen, 13,547 patients (13.6\%) were lost to follow-up, and 8,015 patients $(8.0 \%)$ died during the first 3 years on ART. Figure 2 shows Kaplan-Meier curves of the all-cause mortality for programmes with and without viral load monitoring. Cumulative mortality was slightly higher in non viral load sites in the first 6 months of ART, but curves crossed and separated thereafter, with higher mortality in non viral load sites than in viral load sites $(\mathrm{p}<0.0001)$. When programmes were analyzed separately mortality was highest from month 6 onwards in the two non viral load sites. Figure 3 shows the results of the multi-state model from 6 months after starting ART, based on 84,564 patients with at least 6 months of follow-up. At 3 years 1.3\% (95\% CI 0.9-1.6\%) of patients were on a failing first-line regimen in viral load sites, $9.8 \%(9.1-10.5 \%)$ had switched to second-line ART, 9.2\% (8.5-9.8\%) were lost to follow-up and 4.3\% (3.9-4.8\%) had died. In non viral load sites, more patients were on a failing first-line regimen $(3.7 \%$; 95\% CI 3.6-3.9\%), fewer patients had switched $(2.1 \%, 2.0-2.3 \%)$ and more patients were lost $(15.3 \%, 15.0-$ $15.6 \%)$ or had died $(6.3 \%, 6.0-6.5 \%)$. Estimates for different time points are given in Webtable 3.

Table 2 shows crude and adjusted hazard ratios from multi-state models measuring time from the start of ART to 6 months, and from 6 months to the end of follow-up. Mortality was slightly lower in sites with viral load monitoring during the first six months on therapy (adjusted HR $0.83,95 \%$ CI 0.74-0.92), and substantially lower after the first six months (adjusted HR $0.58,0.50-0.66$ ). Loss to follow-up was similar in the first six months; after six months, however, the risk was lower in viral load sites (adjusted HR 0.53, 0.48-0.58). Patients were more likely to switch to second-line ART in viral load compared to non viral load sites (adjusted HR 4.16,3.57-4.85). Results were similar in sensitivity analyses that omitted one cohort in turn from the analysis (Webtable 4), and similar in the complete-case analysis (Webtable 5).

\section{CD4 cell counts over time}

A CD4 count at baseline and at least one additional CD4 cell count during follow-up were recorded for 8,981 (48.0\%) patients from viral load sites and 42,587 (52.6\%) patients from non viral load sites. The 51,568 patients included in the analysis contributed a total of 257,636 CD4 counts. The median number of CD4 measurements was 4 (interquartile range [IQR] 3-7) in both settings. Figure 4 shows 6-week moving averages of CD4 counts and modelled CD4 trajectories by CD4 count at ART initiation. The trajectories crossed at six months, with less pronounced increases in non viral load sites compared to viral load sites thereafter. Table 3 shows CD4 cell count and viral load data at different time points for viral load and non viral load sites. In viral load sites CD4 cell counts were lower initially, but higher at virologic failure and at the start of second-line ART than in non viral load sites. Three years after starting ART median CD4 cell counts were 425 cells $/ \mu \mathrm{L}$ (IQR 308-582) in viral load sites and 383 cells $/ \mu \mathrm{L}$ (IQR 268-526) in non viral load sites ( $<<0.001$ ). The omission of one cohort in turn did not materially alter results (Webfigure 1).

\section{Non-HIV related mortality}

The expected non-HIV related mortality rate was 0.62 (95\% CI $0.52-0.75)$ per 100 personyears for the South African sites compared to $0.80(0.75-0.85)$ per 100 person-years in Zambia and Malawi. The crude observed mortality from 6 months to 3 years was 2.12 (1.93-2.34) and 3.10 (2.99-3.20) per 100 person-years, respectively. Differences in background mortality could therefore explain about $20 \%$ of the observed mortality difference. Details of calculations are presented in Webtable 6. 


\section{DISCUSSION}

We compared long-term outcomes of ART in four scale-up programmes in RSA, where viral load is routinely monitored, with two programmes from Zambia and Malawi, where only CD4 cell counts are regularly measured. Mortality was somewhat lower initially, and substantially lower after 6 months on ART in viral load compared to non viral load sites. A similar picture was seen for loss to follow-up, with lower rates of loss to follow-up in viral load sites after 6 months. Comparisons of CD4 cell count trajectories were compatible with the findings for mortality, with less pronounced increases in counts in non viral load sites compared to viral load sites after 6 months. Finally, switching to second-line regimens was more frequent in viral load sites. These results were robust in sensitivity analyses, and not driven by a single cohort.

Our study was based on almost 100,000 adult patients from six public sector treatment programmes in three countries. Results should thus be applicable to many other patients in a region heavily affected by HIV. We acknowledge that the treatment programmes included in this study will not be representative for all programmes in the three countries: they are located in urban areas, equipped with electronic medical record systems, and have access to regular CD4 cell determination and second-line therapy [19]. Information on stage of disease and CD4 cell counts was missing in some patients, both in settings with and without routine viral load monitoring. We used multiple imputations to account for the missing information and followed recent guidelines when building these models [20]. We did not examine clinical failure: not all sites systematically collect data on opportunistic infections and diagnostic capabilities and criteria vary between sites. Also, we had no information on adherence or drug resistance.

There is debate on the place of viral load monitoring for ART in resource-limited settings $[21,22]$. A modelling study of long-term ART concluded that the reduction in mortality associated with viral load monitoring is small and cost-effectiveness poor [21]. A randomized trial [23] compared 3-monthly monitoring of CD4 count with viral load monitoring in Thailand and found that over three years switching was somewhat more frequent with CD4 count monitoring compared to viral load monitoring ( $7.2 \%$ versus $5.1 \%$, $\mathrm{p}=0.10)$ and that mortality was similar ( $3.4 \%$ versus $4.3 \%, \mathrm{p}=0.57)$. Modelling studies assume that patients monitored by CD4 counts switch to second-line ART when meeting criteria for immunological failure, and protocols of clinical trials ensure that this is the case. The present study shows that the situation in practice is quite different: few patients switch to second-line ART in programmes relying on CD4 counts to monitor ART in Malawi and Zambia, and many more patients remain on a failing first-line regimen compared to programmes monitoring viral load in RSA. Mortality in patients remaining on failing firstline regimens is high: in a previous study of 11 ART programmes in sub-Saharan Africa we found that mortality at 1 year was $4.2 \%$ (95\% CI $2.2-7.8 \%$ ) in patients who switched to a second-line regimen but $11.7 \%(7.3-18.5 \%)$ in patients who remained on a failing first-line regimen $[5,24]$.

Our results support the notion that routine viral load monitoring contributed to the lower mortality in RSA. First, mortality curves and CD4 trajectories separated after six months, when patients start to switch to second-line therapy and when in viral load sites the first viral load data became available to identify patients for adherence interventions. Data from RSA [25] and Thailand [26] showed that many patients with low-level viraemia $(<1000$ copies/ $\mathrm{ml})$ suppress viral replication after targeted interventions to improve adherence. The monitoring of viral load may thus promote adherence and retention in care [27], in addition to detecting high-level virologic failure requiring second-line therapy. In non viral load sites 
targeted viral load monitoring was used in some patients: $37 \%$ of those starting second line therapy had a viral load measurement.

Second, the higher mortality in programmes without viral load monitoring was not explained by differences in patient characteristics at baseline. The median baseline CD4 cell count was in fact lower in viral load than in non viral load sites. Third, switching to second-line ART occurred later and at lower CD4 cell counts in programmes without viral load monitoring, in line with a previous analysis of ART programmes in lower-income countries [5]. Switching at lower CD4 counts is associated with higher mortality [24, 28]. Finally, at three years, nearly three times as many patients were on a failing first-line regimen in non viral load sites, compared to viral load sites. A study of patients initiating ART in several countries in Asia also found a higher risk of disease progression in patients from sites with fewer than one annual viral load [29]. A meta-analysis of cohort studies demonstrated that in patients treated under WHO guidelines viral load monitoring was associated with reduced probabilities of mutations conferring resistance to NNRTIs, the M184V/I mutation and, thymidine analogue mutations (TAMS) [30].

This was an observational study and other factors than viral load monitoring could explain our results. RSA is a middle-income country with a per capita Gross Domestic Product (GDP) of \$9,332 in 2009 whereas Zambia and Malawi are low-income countries who had per capita GDP of $\$ 1,297$ and $\$ 779$, respectively, in the same year [31]. The ART programmes in the latter two countries lacked access to diagnostic tests and treatments to manage the complications of advanced HIV infection. In RSA resistance testing can be done in programmes linked to an academic department, but not routinely: testing is generally done in the context of research, or in complex cases of second-line failure.

We carefully considered programme attrition: a significant proportion of those lost to follow-up may have died, particularly over the first year of therapy [32]. Analyses of mortality were based on patients remaining in care, and uncounted deaths among patients not returning to clinics could have introduced bias. After six months, patient attrition was almost twice as common in non-viral sites compared to viral load sites: the bias associated with loss to follow-up thus probably means that we underestimated mortality differences. This bias will, however, be modest: fewer than $10 \%$ of patients were lost in the initial 6 months, and between 6 months and 3 years. In this situation the mortality observed among patients retained in care will provide a reasonable estimate of programme-level mortality [33].

In conclusion, over the first three years of ART, mortality, loss to follow-up and CD4 trajectories were more favourable among patients enrolled in programmes in RSA compared to programmes in Malawi and Zambia. These differences may at least partly be explained by more timely switching to second-line ART after virological failure in RSA and better adherence counselling, where monitoring of viral load is routine.

\section{Supplementary Material}

Refer to Web version on PubMed Central for supplementary material.

\section{Acknowledgments}

We thank all study participants and Janne Estill, Leigh Johnson, Franziska Schöni-Affolter, Gilles Wandeler and Robin Wood for helpful discussions and support. The study was supported by the National Institute of Allergy and Infectious Diseases (NIAID), Grant 5U01-AI069924-05 and a PROSPER fellowship to O Keiser supported by the Swiss National Science Foundation (Grant 32333B_131629). Matthew Fox was supported by Award Number K01AI083097 from NIAID. The content is solely the responsibility of the authors and does not necessarily represent the official views of NIAID or the National Institutes of Health. 


\section{References}

1. World Health Organization. Progress Report 2010. Geneva: World Health Organization; 2010. Towards universal access. Scaling up priority HIV/AIDS interventions in the health sector. Available at http://www.who.int/hiv/pub/2010progressreport/en/index.html

2. World Health Organization. 2010 revision. Geneva: World Health Organization; 2010. Antiretroviral therapy for HIV infection in adults and adolescents: recommendations for a public health approach. Available at http://www.who.int/hiv/pub/arv/adult2010/en/index.html

3. Keiser O, MacPhail P, Boulle A, Wood R, Schechter M, Dabis F, et al. Accuracy of WHO CD4 cell count criteria for virological failure of antiretroviral therapy. Trop Med Int Health. 2009; 14:12201225. [PubMed: 19624478]

4. Moore DM, Mermin J, Awor A, Yip B, Hogg RS, Montaner JS. Performance of immunologic responses in predicting viral load suppression: implications for monitoring patients in resourcelimited settings. J Acquir Immune Defic Syndr. 2006; 43:436-439. [PubMed: 17019367]

5. Keiser O, Tweya H, Boulle A, Braitstein P, Schecter M, Brinkhof MW, et al. Switching to secondline antiretroviral therapy in resource-limited settings: comparison of programmes with and without viral load monitoring. AIDS. 2009; 23:1867-1874. [PubMed: 19531928]

6. Egger M, Ekouevi DK, Williams C, Lyamuya RE, Mukumbi H, Braitstein P, et al. Cohort Profile: The international epidemiological databases to evaluate AIDS (IeDEA) in sub-Saharan Africa. Int J Epidemiol. 2011 May 18. [Epub ahead of print].

7. Boulle A, Van Cutsem G, Hilderbrand K, Cragg C, Abrahams M, Mathee S, et al. Seven-year experience of a primary care antiretroviral treatment programme in Khayelitsha, South Africa. AIDS. 2010; 24:563-572. [PubMed: 20057311]

8. Lawn SD, Myer L, Orrell C, Bekker LG, Wood R. Early mortality among adults accessing a community-based antiretroviral service in South Africa: implications for programme design. AIDS. 2005; 19:2141-2148. [PubMed: 16284464]

9. Eshun-Wilson I, Havers F, Nachega JB, Prozesky HW, Taljaard JJ, Zeier MD, et al. Evaluation of paradoxical TB-associated IRIS with the use of standardized case definitions for resource-limited settings. J Int Assoc Physicians AIDS Care. 2010; 9:104-108.

10. Sanne IM, Westreich D, Macphail AP, Rubel D, Majuba P, Van Rie A. Long term outcomes of antiretroviral therapy in a large HIV/AIDS care clinic in urban South Africa: a prospective cohort study. J Int AIDS Soc. 2009; 12:38. [PubMed: 20017918]

11. Beadles WI, Jahn A, Weigel R, Clutterbuck D. Peripheral neuropathy in HIV-positive patients at an antiretroviral clinic in Lilongwe, Malawi. Trop Doct. 2009; 39:78-80. [PubMed: 19299286]

12. Chi BH, Cantrell RA, Mwango A, Westfall AO, Mutale W, Limbada M, et al. An empirical approach to defining loss to follow-up among patients enrolled in antiretroviral treatment programs. Am J Epidemiol. 2010; 171:924-931. [PubMed: 20219765]

13. Stringer JSA, Zulu I, Levy J, Stringer EM, Mwango A, Chi BH, et al. Rapid Scale-up of Antiretroviral Therapy at Primary Care Sites in Zambia: Feasibility and Early Outcomes. JAMA. 2006; 296:782-793. [PubMed: 16905784]

14. Putter H, Fiocco M, Geskus RB. Tutorial in biostatistics: competing risks and multi-state models. Stat Med. 2007; 26:2389-2430. [PubMed: 17031868]

15. Rubin, D. Multiple imputation for nonresponse in surveys. New York: Wiley; 1987.

16. Nash D, Katyal M, Brinkhof MW, Keiser O, May M, Hughes R, et al. Long-term immunologic response to antiretroviral therapy in low-income countries: a collaborative analysis of prospective studies. AIDS. 2008; 22:2291-2302. [PubMed: 18981768]

17. Lopez AD, Mathers CD, Ezzati M, Jamison DT, Murray CJ. Global and regional burden of disease and risk factors, 2001: systematic analysis of population health data. Lancet. 2006; 367:17471757. [PubMed: 16731270]

18. Brinkhof MW, Boulle A, Weigel R, Messou E, Mathers C, Orrell C, et al. Mortality of HIVinfected patients starting antiretroviral therapy in sub-Saharan Africa: comparison with HIVunrelated mortality. PLoS Med. 2009; 6:e1000066. [PubMed: 19399157] 
19. Forster M, Bailey C, Brinkhof MW, Graber C, Boulle A, Spohr M, et al. Electronic medical record systems, data quality and loss to follow-up: survey of antiretroviral therapy programmes in resource-limited settings. Bull World Health Organ. 2008; 86:939-947. [PubMed: 19142294]

20. Sterne JA, May M, Costagliola D, de Wolf F, Phillips AN, Harris R, et al. Timing of initiation of antiretroviral therapy in AIDS-free HIV-1-infected patients: a collaborative analysis of $18 \mathrm{HIV}$ cohort studies. Lancet. 2009; 373:1352-1363. [PubMed: 19361855]

21. Phillips AN, Pillay D, Miners AH, Bennett DE, Gilks CF, Lundgren JD. Outcomes from monitoring of patients on antiretroviral therapy in resource-limited settings with viral load, CD4 cell count, or clinical observation alone: a computer simulation model. Lancet. 2008; 371:14431451. [PubMed: 18440426]

22. Revell AD, Wang D, Harrigan R, Hamers RL, Wensing AM, Dewolf F, et al. Modelling response to HIV therapy without a genotype: an argument for viral load monitoring in resource-limited settings. J Antimicrob Chemother. 2010; 65:605-607. [PubMed: 20154024]

23. Jourdain, G.; Ngo-Giang-Huong, N.; Le Coeur, S.; Traisaithit, P.; Barbier, S.; Techapornroong, M., et al. PHPT-3: A Randomized Clinical Trial Comparing CD4 vs Viral Load ART Monitoring/ Switching Strategies in Thailand. 18th Conference on Retroviruses and Opportunistic Infections; February 27-March 3. 2011; Boston.

24. Keiser O, Tweya H, Braitstein P, Dabis F, MacPhail P, Boulle A, et al. Mortality after failure of antiretroviral therapy in sub-Saharan Africa. Trop Med Int Health. 2010; 15:251-258. [PubMed: 20003034]

25. Orrell C, Harling G, Lawn SD, Kaplan R, McNally M, Bekker LG, et al. Conservation of first-line antiretroviral treatment regimen where therapeutic options are limited. AntivirTher. 2007; 12:8388 .

26. Wilson D, Keiluhu AK, Kogrum S, Reid T, Seriratana N, Ford N, et al. HIV-1 viral load monitoring: an opportunity to reinforce treatment adherence in a resource-limited setting in Thailand. Transactions of the Royal Society of Tropical Medicine and Hygiene. 2009; 103:601606. [PubMed: 19110288]

27. Lawn SD, Bekker LG, Calmy A, Wood R. Monitoring of antiretroviral therapy in low-resource settings. Lancet. 2008; 372:287-288. author reply 289. [PubMed: 18657697]

28. Pujades-Rodriguez M, Balkan S, Arnould L, Brinkhof MA, Calmy A. Treatment failure and mortality factors in patients receiving second-line HIV therapy in resource-limited countries. JAMA. 2010; 304:303-312. [PubMed: 20639564]

29. Oyomopito R, Lee MP, Phanuphak P, Lim PL, Ditangco R, Zhou J, et al. Measures of site resourcing predict virologic suppression, immunologic response and HIV disease progression following highly active antiretroviral therapy (HAART) in the TREAT Asia HIV Observational Database (TAHOD). HIV Med. 2010; 11:519-529. [PubMed: 20345881]

30. Gupta RK, Hill A, Sawyer AW, Cozzi-Lepri A, von Wyl V, Yerly S, et al. Virological monitoring and resistance to first-line highly active antiretroviral therapy in adults infected with HIV-1 treated under WHO guidelines: a systematic review and meta-analysis. Lancet Infect Dis. 2009; 9:409417. [PubMed: 19555900]

31. Hiatt RA, Fireman BH. Serum cholesterol and the incidence of cancer in a large cohort. Journal of Chronic Diseases. 1986; 39:861-870. [PubMed: 3793838]

32. Brinkhof MW, Pujades-Rodriguez M, Egger M. Mortality of patients lost to follow-up in antiretroviral treatment programmes in resource-limited settings: systematic review and metaanalysis. PLoS One. 2009; 4:e5790. [PubMed: 19495419]

33. Egger M, Spycher BD, Sidle J, Weigel R, Geng EH, Fox MP, et al. Correcting mortality for loss to follow-up: a nomogram applied to antiretroviral treatment programmes in sub-Saharan Africa. PLoS.Med. 2011; 8:e1000390. [PubMed: 21267057] 

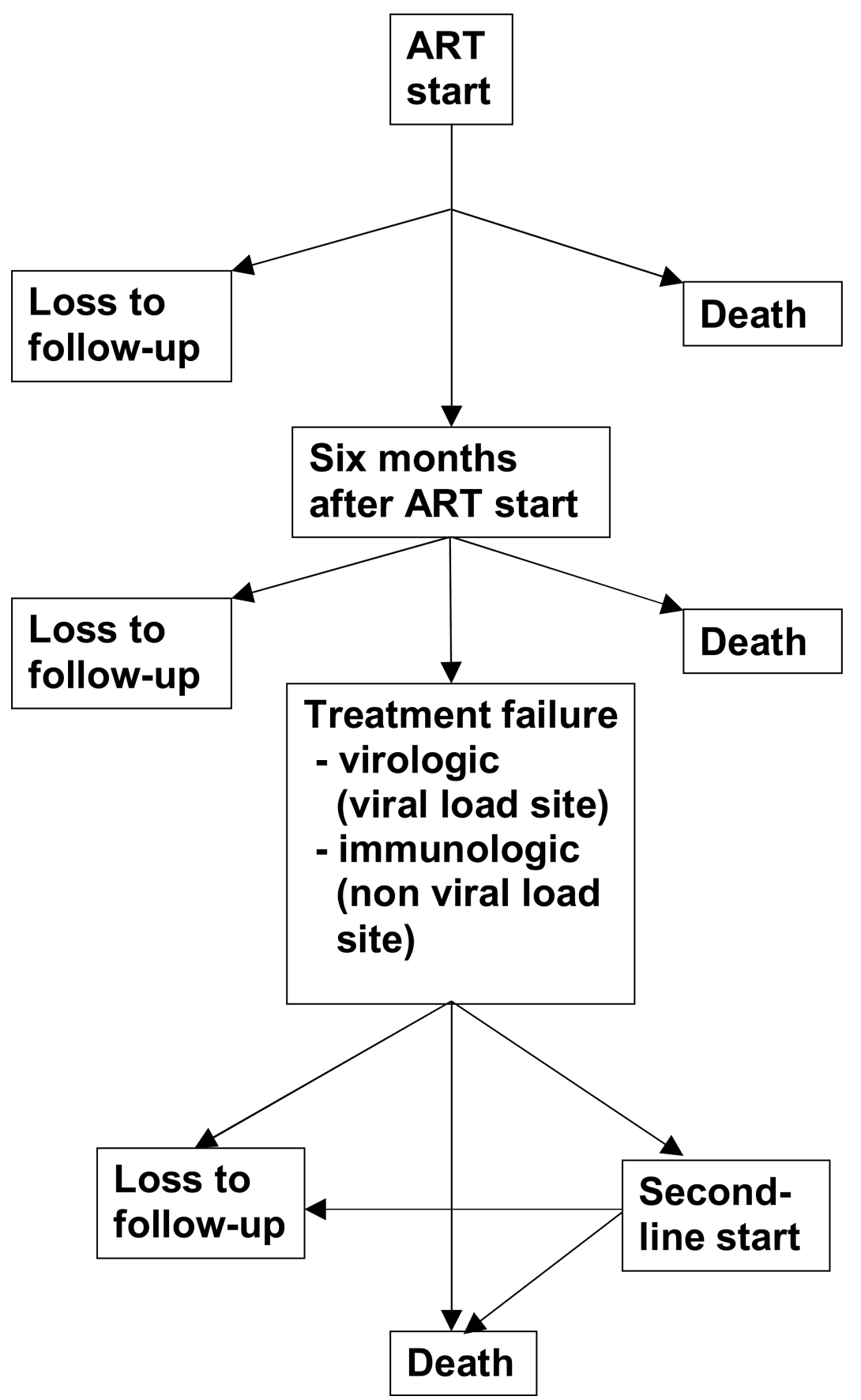

Figure 1.

States and transitions between states in the multi-state model.

$\mathrm{ART}=$ antiretroviral therapy 


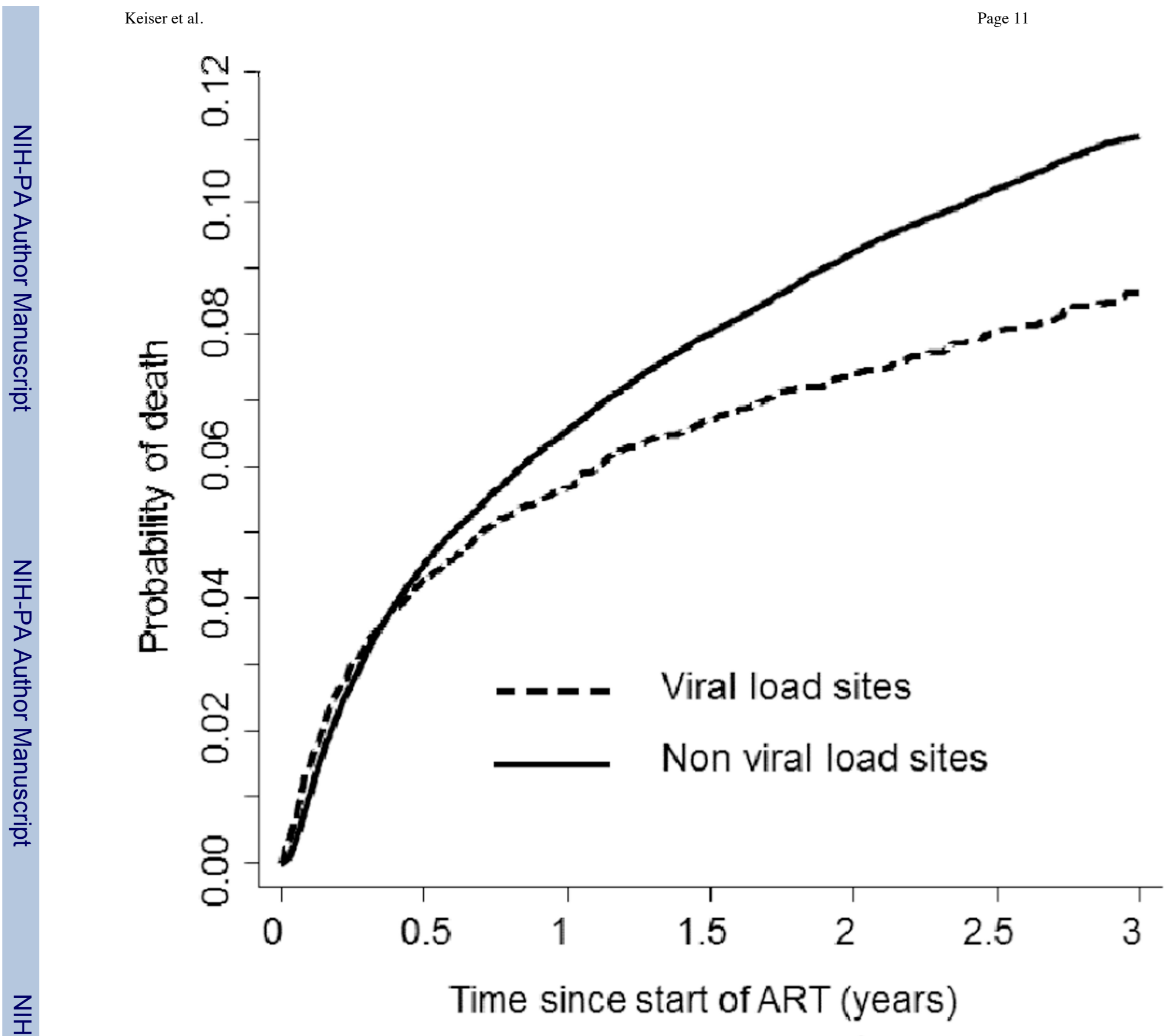

Figure 2.

Cumulative mortality of patients starting antiretroviral therapy in four sites with routine viral load monitoring from the Republic of South Africa and two sites without access to routine viral load monitoring in Malawi and Zambia. 

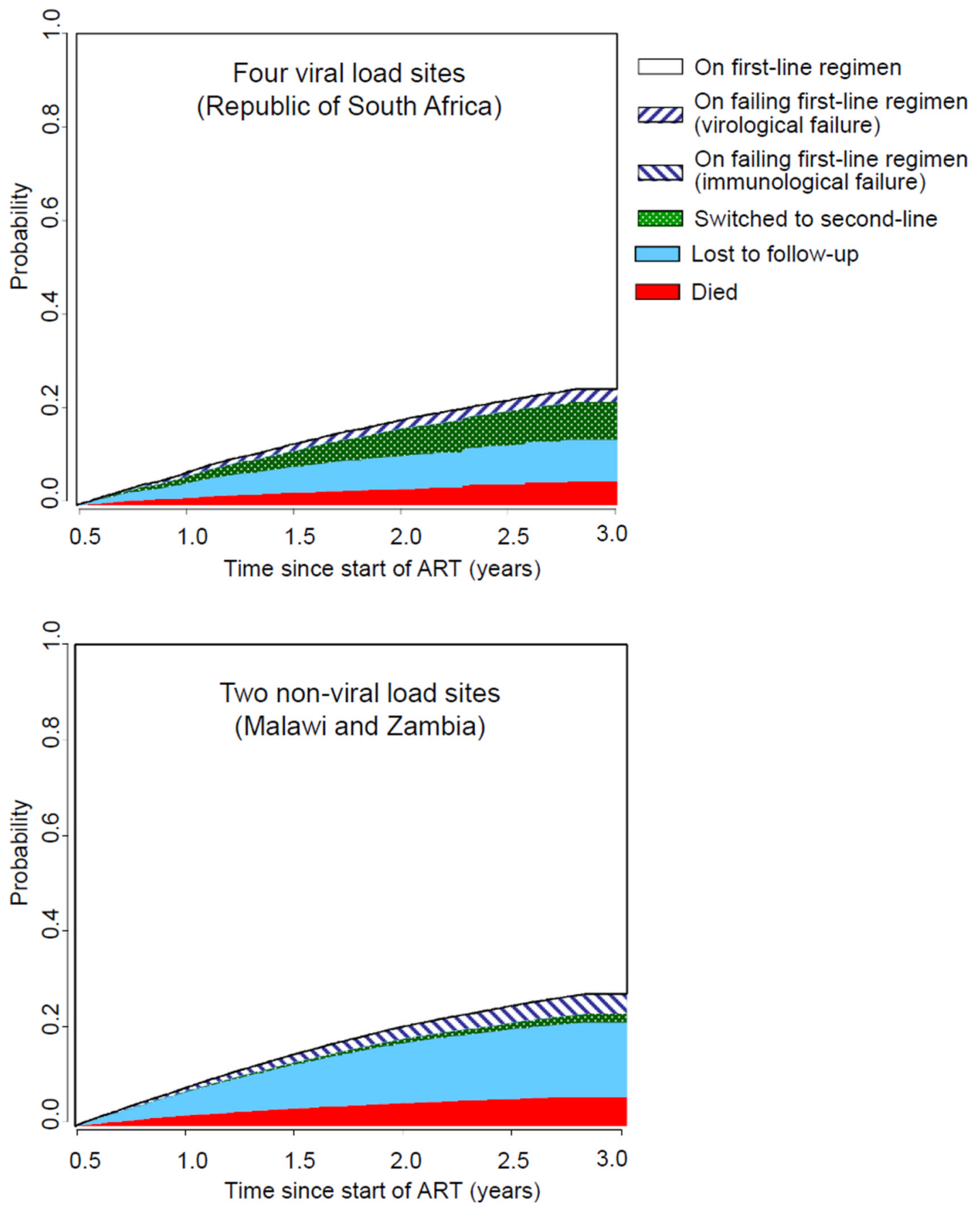

Figure 3.

Cumulative probability of treatment outcomes from 6 months after starting antiretroviral therapy in sites with and without routine viral load monitoring in the Southern Africa IeDEA cohort.

Failure relates to virological failure in viral load sites and immunological failure in non viral load sites and relates to patients who met criteria for failure but have not been switched to second-line therapy. 

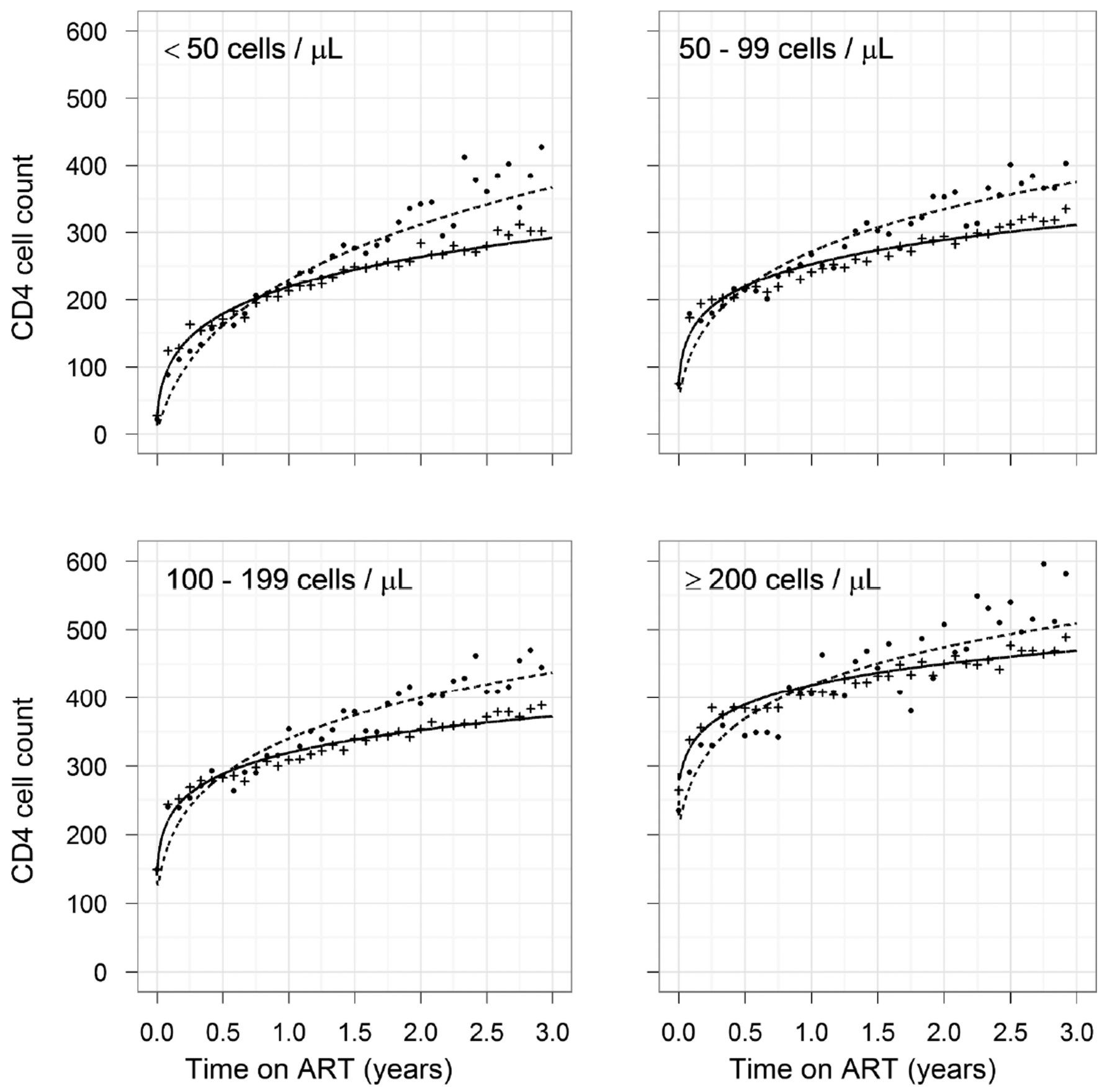

Figure 4.

Evolution of CD4 cell counts from start of antiretroviral therapy (ART) up to three years after start of ART in four sites with routine viral load (Republic of South Africa) and two sites without access to viral load monitoring (Malawi and Zambia).

Lines represent the mean fit of the mixed effect model with $95 \%$ confidence intervals, dots and crosses the moving averages of the observed data. Viral load sites are shown as broken lines and dots, non viral load sites as solid line and crosses. 
Table 1

Patient characteristics in public-sector HIV treatment programmes with and without routine viral load monitoring in the Southern Africa IeDEA cohort.

\begin{tabular}{|c|c|c|}
\hline Variable & $\begin{array}{c}\text { Viral load sites } \\
\text { (Republic of South Africa, } \\
n=18,706)\end{array}$ & $\begin{array}{c}\text { Non-viral load sites } \\
\text { (Zambia and Malawi, } \\
n=80,937)\end{array}$ \\
\hline Women $(\%)$ & $12,291(65.7)$ & $50,036(61.8)$ \\
\hline Median age [IQR] (years) & $34[30-41]$ & $35[30-42]$ \\
\hline \multicolumn{3}{|l|}{ Clinical stage (\%) } \\
\hline Stage available & $18,359(98.1)$ & $77,572(95.8)$ \\
\hline Advanced * & $10,601(57.7)$ & $54,878(70.7)$ \\
\hline \multicolumn{3}{|l|}{ CD4 cell count $($ cells $/ \mu \mathrm{L})$} \\
\hline CD4 count available & $11,880(63.5)$ & $59,939(74.1)$ \\
\hline Median [IQR] & 93 [39-159] & $132[66-203]$ \\
\hline \multicolumn{3}{|l|}{ HIV-1 viral load (copies/ml) } \\
\hline Viral load available (\%) & $7,183(38.4)$ & $839(1.0)$ \\
\hline Median log viral load [IQR] & $5.0[4.5-5.5]$ & \\
\hline$\leq 10,000$ & $805(11.7 \%)$ & $98(11.7 \%)$ \\
\hline $10,001-100,000$ & $2626(36.6 \%)$ & $228(27.2 \%)$ \\
\hline \multicolumn{3}{|l|}{ First-line regimens $(\%)$} \\
\hline $3 \mathrm{TC}$ d4T EFV & $12,231(65.4)$ & $4,970(6.1)$ \\
\hline 3TC ZDV EFV & $954(5.1)$ & $2,756(3.4)$ \\
\hline $3 T C$ d4T NVP & $4,204(22.5)$ & $46,940(58.0)$ \\
\hline 3TC ZDV NVP & $1,217(6.5)$ & $25,419(31.4)$ \\
\hline Other & $100(0.5)$ & $852(1.1)$ \\
\hline \multicolumn{3}{|l|}{ Second-line regimens (\%) } \\
\hline TNV FTC LPV/r & & $817(59.7 \%)$ \\
\hline AZT ddI LPV/r & $657(73.1 \%)$ & \\
\hline $\mathrm{ABC}$ ddI LPV/r & & $231(16.9 \%)$ \\
\hline 3TC AZT LPV/r & $81(9.0 \%)$ & \\
\hline 3TC AZT LPV/r TNV & & $59(4.3 \%)$ \\
\hline 3TC AZT EFV LPV/r & $33(3.7 \%)$ & \\
\hline Other & $128(14.2 \%)$ & $262(19.1 \%)$ \\
\hline
\end{tabular}

Interquartile ranges (IQR) are shown in square brackets and percentages in brackets. ABC, abacavir; ddI, didanosine; EFV, efavirenz; d4T, stavudine; FTC, emtricitabin; LPV, lopinavir; NVP, nevirapine; RTV: boost of ritonavir; 3TC, lamivudine; ZDV, zidovudine

* World Health Organization (WHO) stages III or IV. 


\section{Table 2}

Crude and adjusted hazard ratios for HIV treatment outcomes comparing antiretroviral treatment programmes with and without (reference group) routine viral load monitoring in the Southern Africa IeDEA cohort.

\begin{tabular}{|c|c|c|c|c|}
\hline \multirow[t]{2}{*}{ Outcome } & \multicolumn{2}{|l|}{ Crude analysis } & \multicolumn{2}{|c|}{ Adjusted analysis } \\
\hline & $\begin{array}{c}\text { Crude HR } \\
(95 \% \text { CI })\end{array}$ & $\mathbf{P}$ & $\begin{array}{l}\text { Adjusted*HR } \\
\text { (95\% CI) }\end{array}$ & $\mathbf{P}$ \\
\hline \multicolumn{5}{|l|}{ From start of ART to 6 months } \\
\hline All-cause mortality & $0.97(0.89-1.05)$ & 0.43 & $0.83(0.74-0.92)$ & 0.002 \\
\hline Loss to follow-up & $1.37(1.28-1.48)$ & $<0.001$ & $1.00(0.91-1.11)$ & 0.39 \\
\hline \multicolumn{5}{|c|}{ From 6 months to end of follow-up } \\
\hline All-cause mortality & $0.65(0.58-0.72)$ & $<0.001$ & $0.58(0.50-0.66)$ & $<0.001$ \\
\hline Loss to follow-up & $0.56(0.52-0.60)$ & $<0.001$ & $0.53(0.48-0.58)$ & $<0.001$ \\
\hline Switch to second-line ART & $4.71(4.24-5.24)$ & $<0.001$ & $4.16(3.57-4.85)$ & $<0.001$ \\
\hline
\end{tabular}

Results from multi-state models based on 20 imputed datasets.

* Adjusted for age, sex, first-line regimen, CD4 cell count and clinical stage at start of first line therapy and for age, sex, first-line regimen and CD4 cell count at 6 months. 


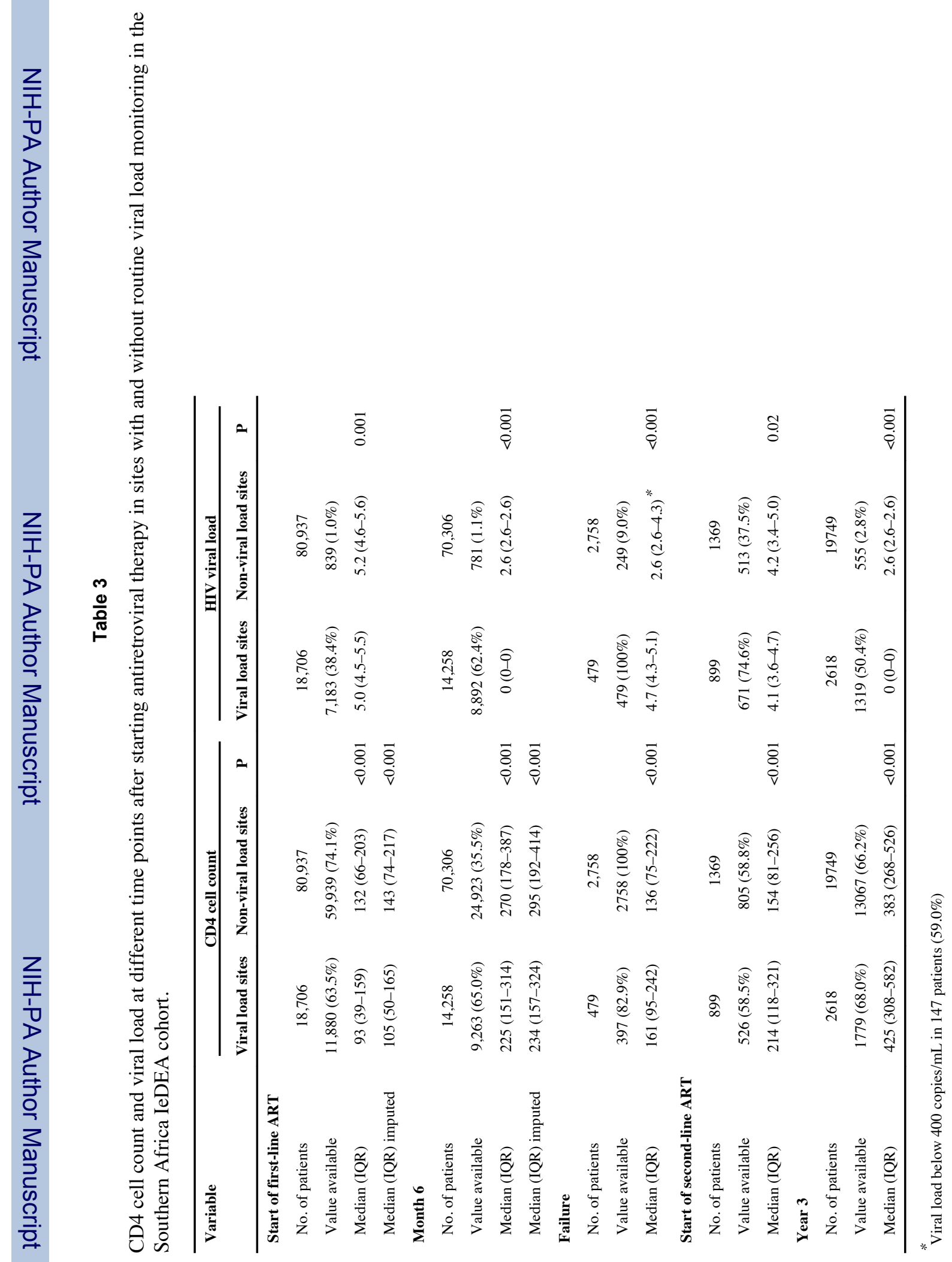

